\title{
Corrigendum to "Characterization of Rice Husk Fiber-Reinforced Polyvinyl Chloride Composites under Accelerated Simulated Soil Conditions"
}

\author{
Lei Wang $(\mathbb{D}$ and Chunxia He \\ College of Engineering, Nanjing Agricultural University, Nanjing 210031, China \\ Correspondence should be addressed to Chunxia He; chunxiahe@hotmail.com
}

Received 4 December 2020; Accepted 4 December 2020; Published 15 January 2021

Copyright (C) 2021 Lei Wang and Chunxia He. This is an open access article distributed under the Creative Commons Attribution License, which permits unrestricted use, distribution, and reproduction in any medium, provided the original work is properly cited.

In the article titled "Characterization of Rice Husk FiberReinforced Polyvinyl Chloride Composites under Accelerated Simulated Soil Conditions" [1], the authors identified an error in the reported cut-off period, which should be corrected from 7 days to 30 days. Additionally, an error occurred in the process of exporting and drawing the infrared data, which led to an error in the 14-day aging time curve of Figure 3. The corrected Figure 3 is shown below.

The authors repeated the experiment to verify the conclusion, and the results are included in the supplementary materials.

A related article was not cited in the original publication. Article [1] used orthogonal experiments to determine the soil aging conditions and studied the performance changes before and after aging of rice husks (100 meshes) and PVC (100 meshes) composites. Article [2] focused on the optimization of rice husk/PVC formulations.

Since the second paper was an extension of the first paper, the authors compared the data from [1] to prove the conclusions. In article [2], the formula of MSRC was a particle size ratio of mixed-particle-size fibers of $80: 120$, mass ratio of mixed-particle-size fibers of $7: 3$, and mass ratio of wood-to-plastic of $4: 6$. The microstructure of RHRC ( 0 day and 21 days) shown in Figures 3(a) and 3(b) of article [2] corresponds to Figures 2(a) and 2(b) in article [1]. 


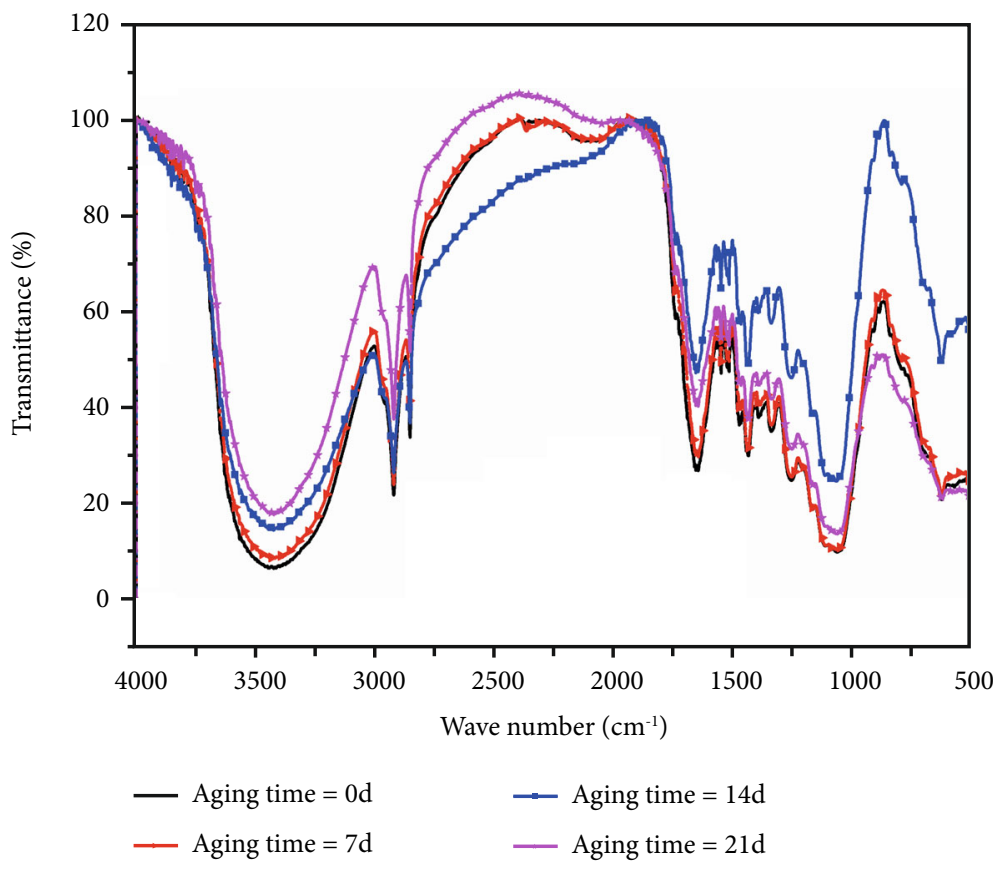

FIGURE 3: FTIR spectra of the composites.

\section{References}

[1] L. Wang and C. He, "Characterization of rice husk fiberreinforced polyvinyl chloride composites under accelerated simulated soil conditions," International Journal of Polymer Science, vol. 2019, Article ID 5409821, 9 pages, 2019.

[2] L. Wang and C. He, "Effects of rice husk fibers on the properties of mixed-particle-size fiber-reinforced polyvinyl chloride composites under soil accelerated aging conditions," Journal of Engineered Fibers and Fabrics, vol. 14, 2019. 